\title{
Right frontal lobe slow frequency repetitive transcranial magnetic stimulation (SF r-TMS) is an effective treatment for depression: a case-control pilot study of safety and efficacy
}

\author{
Daniel L Menkes, Peter Bodnar, Roderick A Ballesteros, Michael R Swenson
}

\begin{abstract}
Major depression may result from decreased left frontal lobe function with respect to the right. Fast frequency repetitive transcranial magnetic stimulation (FF r-TMS) excites the underlying cortex whereas slow frequency repetitive transcranial magnetic stimulation (SF r-TMS) causes cortical inhibition. Left frontal FF r-TMS attenuates major depression whereas the inhibitory effects of right frontal SF r-TMS are unknown. This study tested the hypothesis that right frontal SF r-TMS would treat depressed patients with minimal effect on controls.

A psychiatrist administered the Beck depression inventory and Hamilton D depression rating scales to eight depressed patients and six controls before and after the treatment protocol. Eight sessions of 100 right frontal lobe SF r-TMS were given at motor threshold and $0.5 \mathrm{~Hz}$ over a 6 week period.
\end{abstract}

No adverse outcomes were noted in Department of
Neurology, University of Louisville, 500 South Presyon Street, HSC 113, Louisville, KY 40202, USA

D L Menkes

M R Swenson

60th MDOS/SGOMU, 101 Bodin Circle, Travis AFB, CA 94535, USA

R A Ballesteros

249 Ballindine Drive, Vacaville, CA 95688, USA

P Bodnar

Correspondence to: Dr D L Menkes,

Department of Neurology, University of Louisville, 500 South Presyon Street, HSC 113, Louisville, KY 40202, USA

Received 17 July 1998 and in final form

23 December 1998

Accepted 27 January 1999 pulse (TMS) or as a train of repetitive stimuli (r-TMS) induces an electric field in the underlying cortex. r-TMS is further subdivided into fast frequency (FF r-TMS) for rates $>1 \mathrm{~Hz}$ and slow frequency (SF r-TMS) for rates $<1 \mathrm{~Hz}$. Both frequencies induce immediate cortical suppression but only FF r-TMS produces subsequent cortical excitation. ${ }^{3}$

Mood disorders may result from a relative imbalance of frontal lobe function wherein depression occurs with a hypofunctioning left frontal lobe whereas mania may result from the opposite situation. ${ }^{4}$ Additional evidence supporting this paradigm is the finding of reduced left frontal lobe glucose metabolism in depressed patients. ${ }^{5}$ Furthermore, net cortical excitation with left frontal FF r-TMS was effective in treating depression whereas right frontal lobe FF r-TMS treated mania. ${ }^{6}$ However, FF r-TMS has side effects ranging from patient discomfort to seizure induction. ${ }^{8}$

By contrast, SF r-TMS is neither painful nor epileptogenic. An open, uncontrolled trial conducted subsequent to this one showed therapeutic efficacy of SF r-TMS in depressed and schizophrenic patients. ${ }^{9}$ This study lent further credence to our hypothesis that frontal lobe imbalance could be addressed either by excitation of the hypofunctioning cortex or suppression of its contralateral homologue. This study was conducted to validate this concept in a pilot study of normal subjects and depressed patients who underwent an identical right frontal lobe SF r-TMS experimental protocol.

\section{Methods}

Eight otherwise healthy persons who met DSM-IV clinical criteria for dysthymia or unipolar depression without psychotic features and six healthy controls completed the protocol. Both groups were instructed to abstain from any mood altering substances or medications except those prescribed. Depressed patients were given the option of adjunctive medical therapy. Our psychiatrist (PB) administered the Hamilton-D and Beck depression inventory scales before and within 2 weeks after the TMS protocol. Motor threshold was determined as the percentage output of a Cadwell 
Summary of results

\begin{tabular}{|c|c|c|c|c|}
\hline & \multicolumn{2}{|c|}{ Controls $(n=6)$} & \multicolumn{2}{|c|}{ Depressed $(n=8)$} \\
\hline & Mean & Range & Mean & Range \\
\hline Age & 32.3 & $22-36$ & 33.3 & $24-40$ \\
\hline No of males & 4 & & 3 & \\
\hline Handedness (No left) & 2 & & 3 & \\
\hline TMS threshold & 48.3 & $45-55$ & 49.4 & $45-55$ \\
\hline Beck entry score & 0.5 & $0-2$ & 10.6 & $3-20$ \\
\hline Beck ending score & 0.2 & $0-1$ & 6 & $0-20$ \\
\hline Ham-D entry score & 0.7 & $0-2$ & 18.4 & $6-33$ \\
\hline Ham-D ending score & 0.8 & $0-4$ & 10.6 & $4-31$ \\
\hline
\end{tabular}

MES-10 stimulator that produced an identical motor evoked potential elicited in the dominant hand in three of six consecutive trials. The right dorsal frontal lobe was located by mirroring the technique of Pascual-Leone. ${ }^{10}$ Each right frontal lobe stimulation session consisted of five sets of 20 stimuli given at $0.5 \mathrm{~Hz}$ separated by a 1 minute rest period at motor threshold. All subjects underwent eight sessions within a 6 week period with no more than 1 week between treatments and never more than two treatments a week.

Statistical analysis was performed by a statistician blinded to the hypothesis. Normally distributed data were analysed with a $t$ test. Non-normally distributed data were assessed with the non-parametric Mann-Whitney $U$ test. A one way analysis of variance (ANOVA) test was used to reject the null hypothesis that the improvement in the depressed patients could be attributed solely to the effects of time.

\section{Results}

No significant differences were noted between the control and depressed groups with respect to age, sex, threshold, or handedness ( $p>0.05)$. These data are summarised in the table. No depressed patient reported any adverse effects. Two controls reported one episode of a transient tension headache. One female subject in each group was excluded for protocol violation. The control later admitted to a history of depression whereas the experimental subject became pregnant.

The study entry scores were significantly different between the normal and depressed groups on both rating scales $(p<0.01)$. No changes were noted in the normal subjects between study entry and completion scores on either scale $(p>0.05)$. An ANOVA test on the depressed patients' scores was able to reject the null hypothesis that the improvement was due solely to time $(\mathrm{p}>0.05)$. The depressed patients showed a statistically significant improvement on both scales; Beck depression inventory $(p<0.05)$ and Hamilton-D $(p<0.02)$. Four depressed patients requested adjunctive medical therapy with selective serotonin reuptake inhibitors (three used paroxetine, one used sertraline). One person using paroxetine was also given adjunctive treatment with imipramine. When depressed subgroup analysis of patients was undertaken, the TMS alone group had a marginal but insignificant trend of greater improvement $(p>0.05)$. For all depressed patients, there was a significant difference between the Beck depression inventory and
Hamilton-D scores before and after the treatment $(\mathrm{p}<0.05)$.

\section{Discussion}

This study shows a statistically significant antidepressant effect of right frontal SF r-TMS limited to depressed patients, with no adverse effects in either group. These results support the frontal lobe imbalance paradigm of unipolar depression. Although Hamilton-D scores showed greater statistical significance than the Beck depression inventory, the fact that both scores improved in the same direction suggests a true effect rather than observer bias.

To our knowledge, this is the first controlled antidepressant trial of right frontal SF r-TMS. Unlike the dysphoria reported by controls receiving FF r-TMS, our controls experienced no such adverse effects. ${ }^{11}$ FF r-TMS may cause greater initial cortical inhibition than SF r-TMS, which may increase the risk of adverse effects. In addition to the lack of adverse effects on controls, SF r-TMS is less expensive, safer, and easier to administer.

Despite these encouraging results, several caveats are in order. All depressed patients were treated on an outpatient basis. As such, the effects of SF r-TMS on severe, psychotic, and bipolar depression are still unknown. This trial did not compare the efficacy of SF r-TMS versus FF r-TMS or ECT. Thus, we concur with previously published recommendations that TMS should neither be employed as a first line treatment for depression nor substituted for electroconvulsive therapy in drug resistant depression. ${ }^{12}$ An exception might be made for those at high risk for general anaesthesia. Despite these precautions, SF r-TMS may prove to be an alternative treatment for those who are unwilling or unable to undergo medical therapy or electroconvulsive therapy. The results of this study justify further research into this novel and promising technique.

Disclaimer: The views expressed in this article represent those of the authors and should not be construed to represent those of the United States Air Force or the Department of Defense. All subjects who participated in this study gave their voluntary informed consent. All studies were conducted in accordance with local IRB policy and with United States Air Force Instructions concerning research on human subjects.

1 Weissman MM, Bland RC, Canino GJ, et al. Cross-national epidemiology of major depression and bipolar disorder. ҰAMA 1996;276:293-9.

2 Segman RH, Shapira B, Gorfine M, et al. Onset and time course of antidepressant action: psychopharmacologic implications of a controlled trial of electroconvulsive implications of a controlled trial of electroconv

3 George MS. TMS/rTMS in psychiatry. Presented at the Harvard Medical School's Course on Transcranial Magnetic Harvard Medical School's Course on Transcranial Magnetic
Stimulation; December 3, 1997; Boston. Boston, MA: Harvard Medical School, 1997.

4 Cummings JL. The neuroanatomy of depression. F Clin Psychiatry 1993;5:14s-20s.

5 Hirano N, Mori E, Ishii K, et al. Frontal lobe hypometabolism and depression in Alzheimer's disease. Neurology 1998;50:380-3

6 George MS, Wassermann EM, Kimbrell TA, et al. Am $\mathcal{F}$ Psychiatry 1997;154:1752-6.

7 Grisaru N, Chudakov B, Yaroslavsky Y, et al. Transcranial magnetic stimulation in mania: a controlled study. $A m$ f Psychiatry 1998;155:1608-10.

8 Wassermann EM. Risk and safety of repetitive transcranial magnetic stimulation: report and suggested guidelines from the International Workshop on the Safety of Repetitive Transcranial Magnetic Stimulation. Electroencephalogr Clin Neurophysiol 1998;108:1-16.

9 Feinsod M, Kreinin B, Chistyakov A, et al. Preliminary eviFeinsod M, Kreinin B, Chistyakov A, et al. Preliminary evi-
dence for a beneficial effect of low frequency, repetitive 
transcranial magnetic stimulation in patients with major transcranial magnetic stimulation in patients with major dep.

10 Pascual-Leone A, Rubio B, Pallardo F, et al. Beneficial effect of transcranial magnetic stimulation of the left dorsofrontal prefrontal cortex in drug-resistant depression. Lancet 1996;348:233-7.
11 Pascual-Leone A, Catala MD, Pascual A. Lateralized effect of rapid-rate transcranial magnetic stimulation of the prefrontal cortex on mood. Neurology 1996;46:499-502.

2 Markwort S, Cordes P, Aldenhoff J. Transcranial magnetic stimulation as an alternative to electroshock therapy in treatment resistant depressions. A literature review. Fortschr Neurol Psychiatr 1997;65:540-9.

\section{Severe necrotising cutaneous lesions complicating treatment with interferon $\beta-1 \mathrm{a}$}

Subgroups of interferon- $\alpha$ (natural interferon- $\alpha$, recombinant interferon $\alpha-1$, recombinant interferon $\alpha-2 \mathrm{a}$, recombinant interferon $\alpha-2 b),{ }^{1-3}$ recombinant interferon $\beta-1 b$, and recombinant interferon- $\gamma$ are known to produce severe necrotising cutaneous reactions. ${ }^{4}$ A 58 year old woman with an 11 year history of chronic inflammatory demyelinating polyneuropathy (CIDP) was treated by recombinant interferon $\beta$-1a (12 million units) subcutaneously three times a week following the protocol of an open label multicentre study based on the description of Choudhary et al. ${ }^{5}$ Erythematous patches and local pain appeared at the injection sites on both thighs and on the abdomen in the first week of treatment. The areas on the abdomen became violaceous with black, necrotic ulcers during the 3rd month of treatment (figure, A). Biopsy 3 months after the beginning of the treatment disclosed interstitial and perivascular lymphocytic infiltrates in the upper dermis (figure, B). Deeper sections showed focal thrombosis of a vessel. There was also a discrete lymphocytic infiltate in the subcutis. The areas healed after discontinuation of the injections in the abdomen while continuing applications in the thighs with a lower dose ( 6 million units). In the 5 th month the normal dose of 12 million units was reinstituted and the patient reported the reappearance of slightly more pain and slightly bigger erythematous patches in the thighs. Now (1

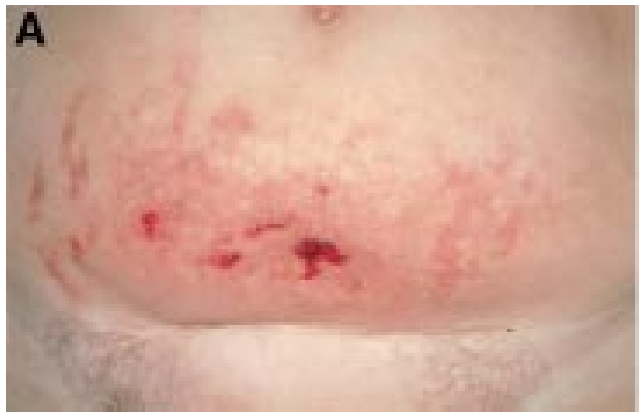

year after the beginning of the treatment) the patient continues the interferon injections without pain and without further dermatological complications. To our knowledge such severe cutaneous reactions have not been reported with the recombinant interferon $\beta-1 \mathrm{a}$ preparation that is recommended for subcutaneous administration. We postulate that all types of interferon can cause necrotising cutaneous lesions. The pathogenesis of these cutaneous reactions due to interferon despite different hypotheses is unknown.

ALEXANDER J RADZIWILL
Department of Neurology
SALOME COURVOISIER
Department of Dermatology, University of Basel,
Basel, Switzerland

Correspondence to: Dr Alexander J Radziwill, Department of Neurology, Kantonsspital, CH-4031 Basel, Switzerland.

1 Oeda E, Shinohara K. Cutaneous necrosis caused by injection of $\alpha$-interferon in patient with chronic myelogenous tion of $\alpha$-interferon in patient with chron

2 Ezekowitz RA, Mulliken JB, Folkman J. Interferon $\alpha-2 \mathrm{a}$ therapy for life-threatening hemangiomas of infancy. $N$ Engl $\mathscr{F}$ Med 1992;326:1456-63.

3 Cnudde F, Gharakhanian S, Luboinski J, et al. Cutaneous ocal necrosis following interferon injections. Int $f$ Dermatol 1991;30:147.

4 Sheremata WA, Taylor JR, Elgart GW. Severe necrotizing cutaneous lesions complicating treatment with interferon beta-1b. N Engl F Med 1995;332:1584.

5 Choudhary PP, Thompson N, Hughes RA. Improvement following interferon $\beta$ in chronic inflammatory demyelinating polyradiculoneuropathy. $\mathcal{F}$ Neurol 1995;242:252-3.

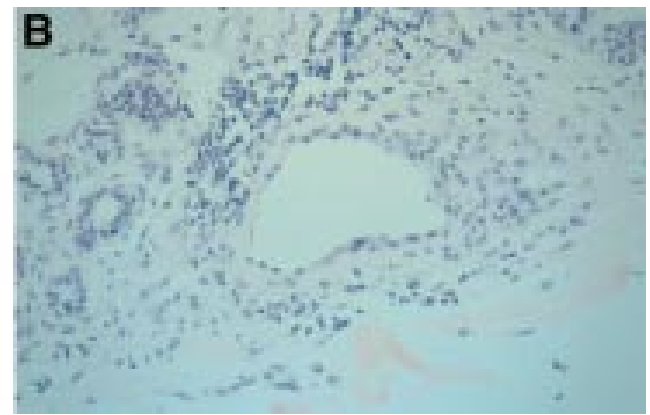

